\title{
NEW Antistatic Chamber: small, solid and effective
}

Aspen is proud to announce the launch of VORTEX ${ }^{\circledR}$, an innovative aluminium antistatic holding chamber with 'cyclone twist' principle, as an addition to our respiratory portfolio.

VORTEX ${ }^{\circledR}$ inhalation aid is suitable in providing: ${ }^{[1]}$

- High lung deposition, low throat deposition

- High dosage consistency

- Disinfectable, ergonomic SmartTouch masks

Reference: 1. Laube BL, Janssens HM, de Jongh FHC, et al. What the pulmonary specialist should know about the new inhalation therapies. Eur Respir J 2011;37:1308-1331.

\section{6) aspen ...n...... Healthcare. We Care.




\section{Rivaroxaban reduces length of hospital stay in patients with symptomatic venous thromboembolism}

The phase III EINSTEIN deep vein thrombosis (DVT) and EINSTEIN pulmonary embolism (PE) trials demonstrated the potential of oral rivaroxaban (Xarelto, Bayer) - $15 \mathrm{mg}$ twice daily for 21 days, followed by $20 \mathrm{mg}$ once daily - for the treatment of venous thromboembolism (VTE), a term that embraces DVT and PE. A subsequent study by van Bellen et al.. ${ }^{[1]}$ published in Current Medical Research and Opinion in 2014, was undertaken to assess the length of initial hospitalisation in patients presenting with either symptomatic DVT or PE using hospitalisation records from these trials.

The authors found that overall 52\% of EINSTEIN DVT patients and $90 \%$ of EINSTEIN PE patients were admitted to hospital. The proportion of hospitalised DVT patients with a length of stay 5 days or fewer, receiving rivaroxaban, was $54 \%$ compared with $31 \%$ for those receiving enoxaparin/vitamin K antagonist (VKA), the current standard of care for the treatment of patients with symptomatic DVT and PE. For patients with PE, the corresponding values were $45 \%$ and $33 \%$. Stays of 6 - 10 days were observed in $29 \%$ of rivaroxaban-treated patients compared with $45 \%$ for enoxaparin/VKA-treated patients for DVT. For patients with PE, these values were $39 \%$ and $46 \%$ in the rivaroxaban and enoxaparin/ VKA groups, respectively. Overall, length of stay was significantly shorter in the rivaroxaban group, compared with the enoxaparin/VKA group across all analyses performed $(p<0.0001)$.

VTE is associated with significant morbidity and mortality and therefore carries a considerable healthcare burden. Rivaroxaban is as effective as enoxaparin/VKA for the treatment of acute symptomatic DVT or PE, with the additional benefit of significantly reducing the period of hospitalisation in patients being treated for an initial DVT or PE. 'Coupled with improved patient treatment satisfaction and no requirement for routine monitoring or dose adjustment, this presents strong advantages for treating patients with VTE with rivaroxaban,' the authors wrote. They concluded that a single-drug regimen with rivaroxaban may reduce the burden on healthcare systems and patients by providing effective and well-tolerated treatment. 'The convenience of a single-drug approach with oral rivaroxaban has the potential to allow discharge based on a patient's clinical condition and to facilitate the transition from in-hospital to outpatient care. [...] However, assessment of patient risk is still warranted to identify candidates who can safely receive outpatient treatment, and patient monitoring is essential to ensure adherence to the specified dosing regimen.'

\section{Reference}

1. van Bellen B, Bamber L, Correa de Carvalho F, et al. Reduction in the length of stay with rivaroxaban as a single-drug regimen for the treatment of deep vein thrombosis and pulmonary embolism. Curr Med Res Opin 2014; 30(5):829-837. [http://dx.doi.org/10.1185/03007995.2013.879439]

For full prescribing information, refer to the package insert approved by the Medicines Regulatory Authority (MCC).

PHARMACOLOGICAL CLASSIFICATION: A.8.2 Anticoagulants.

S4 XARELTO 10. Reg. No.: 42/8.2/1046. Each film-coated tablet contains rivaroxaban $10 \mathrm{mg}$.

INDICATION: Prevention of VTE in patients undergoing major orthopaedic surgery of the lower limbs.

S4 XARELTO 15 and XARELTO 20 . Reg. No.: 46/8.2/0111 and 46/8.2/0112. Each film-coated tablet contains rivaroxaban $15 \mathrm{mg}$ or $20 \mathrm{mg}$, respectively. INDICATIONS: Prevention of stroke and systemic embolism in patients with non-valvular atrial fibrillation; Treatment of DVT and for the prevention of recurrent DVT and PE; Treatment of PE and for the prevention of recurrent PE and DVT.

Bayer (Pty) Ltd, Co. Reg. No.: 1968/011192/07, 27 Wrench Road, Isando, 1609. Tel: 0119215044 Fax: 0119215041.

L.ZA.GM.06.2014.1007
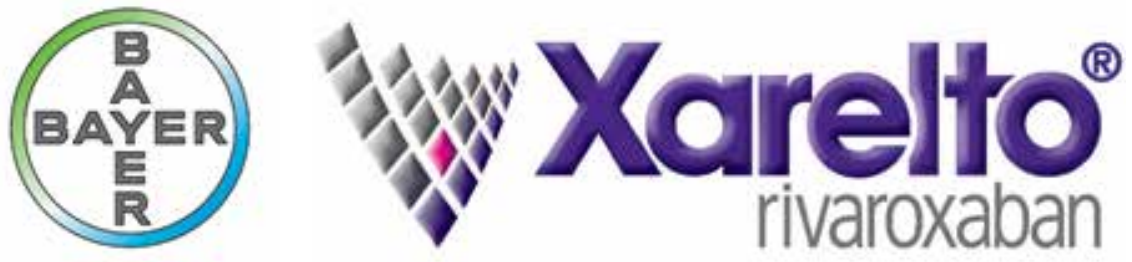

๑ Bayer HealthCare Pharmaceuticals June 2014 